\title{
Evaluación productiva de ovejas y corderos bajo pastoreo con y sin suplementación estratégica
}

\section{Evaluation of sheep and their lamb's productive performance with or without strategic supplementation under grazing conditions}

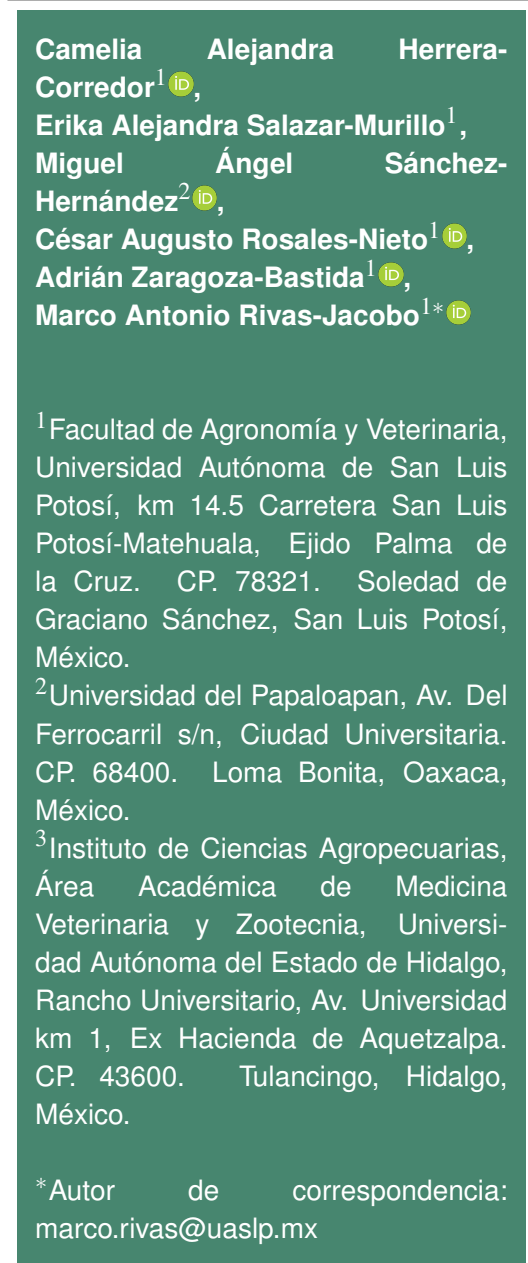

Artículo científico

Recibido: 06 de marzo 2021

Aceptado: 06 de octubre 2021

Como citar: Herrera-Corredor CA, Salazar-Murillo EA, SánchezHernández MA, Rosales-Nieto CA, Zaragoza-Bastida A, Rivas-Jacobo MA (2021) Evaluación productiva de ovejas y corderos bajo pastoreo con y sin suplementación estratégica. Ecosistemas y Recursos Agropecuarios Núm. Esp. II: e2972. DOI: 10.19136/era.a8nll.2972
RESUMEN. Se evaluó el comportamiento productivo de ovejas en pastoreo con y sin suplementación en épocas críticas y el comportamiento de los corderos en la Facultad de Agronomía y Veterinaria de la Universidad Autónoma de San Luis Potosí, a $22.23^{\circ} \mathrm{LN}$ y a $100.85^{\circ} \mathrm{LO}$, a $1835 \mathrm{msnm}$ en clima seco templado. Se utilizaron 24 hembras ovinas de la raza Rambouillet, con un peso promedio de 55 $\mathrm{kg}$ en praderas irrigadas de festuca alta (Festuca arundinaceae). Se utilizaron tres tratamientos, 1) solo pastoreo, 2) $300 \mathrm{~g} \mathrm{día}^{-1}$ de suplemento (rastrojo + pollinaza $10 \%$ + zeolita $5 \%$ ) por oveja. y 3) $400 \mathrm{~g} \mathrm{día}^{-1}$ de suplemento (rastrojo + pollinaza $20 \%+$ zeolita $5 \%$ ) por oveja. A las ovejas se le midió el Peso al empadre (PE), al parto (PP) y al destete (PD), Condición corporal al empadre (CCE), parto (CCP) y destete (CCD); a los corderos se le midió Peso al nacimiento del cordero (PNC) y destete (PDC), además Ganancia de Peso de Hembras (GPH), y Ganancia diaria de peso de corderos (GDPC). Se utilizó un modelo estadístico completamente al azar. Peso, ganancia de peso y condición corporal de hembras mostraron diferencias significativas $(p<0.05)$ por la suplementación estratégica al suministrar mayores nutrientes en las etapas de empadre, último tercio de gestación y lactancia, lo que se reflejó en mayores pesos de los corderos al nacimiento y destete. Por lo que se concluye que las productividades de hembras estuvieron influenciadas por el suplemento estratégico a base de rastrojo, gallinaza y zeolita.

Palabras clave: Gallinaza, peso, ovinos, reproducción, zeolita.

ABSTRACT. We evaluated the sheep and their lamb's productive performance with or without supplementation under grazing conditions at the Agronomy and Veterinary Faculty of the Autonomous University of San Luis Potosi $\left(22.23^{\circ} \mathrm{N}\right.$ y a $100.85^{\circ} \mathrm{W} ; 1835 \mathrm{masl}$ ) We used 24 Rambouillet ewes with an average weight of 55 $\mathrm{kg}$ and they were grazing in Festuca arundinaceae. Sheep were distributed in three treatments 1) only grazing, 2) grazing and supplemented with $300 \mathrm{~g} / \mathrm{d} / \mathrm{ewe}$ of stubble + chicken manure $10 \%$ + zeolite $5 \%, 3)$ grazing and supplemented with $400 \mathrm{~g} / \mathrm{d} / \mathrm{ewe}$ of stubble + chicken manure $20 \%$ + zeolite $5 \%$. Mating live body weight (MLWT), body live weight change (LWTC), lambing live body weight (LLWT), and weight at weaning (MWWT), body condition score at mating (MBCS), lambing (LBCS), and weaning (WBCS) of the dams were recorded, and birth weight (BWT), daily live weight (LWG), and weaning weight (WWT) of the progeny were recorded as well. The data were analyzed with a completely randomized design. Maternal weight and body condition score variables differed among treatments $(p<0.05)$ due to the nutritional supplementation at mating, late gestation, and lactation. Consequently, the lambs from the supplemented treatments were heavier at birth and weaning. We concluded that the sheep productivity was influenced by the strategic supplement based on stubble, chicken manure and zeolite.

Key words: Sheep, reproductive, weight, chickpea, zeolite. 


\section{INTRODUCCIÓN}

La producción de ovinos en México en su mayoría se realiza bajo pastoreo en forma extensiva, con agravantes en la nutrición de los animales en las estaciones de secas de invierno y primavera (Ben y Nefzaoui 2003). Para mejorar la nutrición de ovinos en pastoreo se recomienda el uso de alimentos que complementen el valor nutritivo de la dieta durante la temporada de sequía y épocas críticas, que podría favorecer ganancias de peso de $100 \mathrm{~g} \mathrm{día}^{-1}$ (Galaviz et al. 2011). Las dietas de cabras y ovejas basadas en forrajes de baja calidad y residuos de cosecha, deben incluir una suplementación de proteína con el fin de incrementar el consumo y la digestibilidad (Grajales et al. 2011), y más si están gestantes ya que se demanda mayor cantidad de nutrimentos para el desarrollo del feto, ya que este adquiere hasta un $75-80 \%$ de su peso corporal antes del nacimiento (Hawken et al. 2012). El objetivo de la suplementación durante el empadre aporta dos beneficios; animales que no habían concebido durante el primer ciclo estral de la temporada, se mantendrán en buena condición corporal con valores de tres o más y animales que lograron concebir, tienen un menor riesgo de muerte embrionaria temprana, dando como resultado una depresión en el rendimiento reproductivo de las ovejas manejadas bajo sistema extensivo (Fthenakis et al. 2012). La suplementación en los primeros dos tercios de gestación tiene el objetivo de mantener a las borregas con su peso, por lo que se pueden alimentar con rastrojos y forrajes de mediana calidad y minerales. Sin embargo, en el último tercio de gestación cuando los requerimientos aumentan, sobre todo si son de partos múltiples, en esta etapa ocurre el $70 \%$ del crecimiento del feto. En la última etapa de gestación, el feto adquiere un $75-80 \%$ de su peso corporal futuro al nacimiento (Fthenakis et al. 2012), por lo que los requerimientos de energía y proteína (De 10 a 14\% PC) en las ovejas preñadas aumentan drásticamente, esto se justifica frente a las necesidades de desarrollo fetal y a la necesidad de preparar la glándula mamaria para la producción del calostro (Hawken et al. 2012, Rosales et al. 2015). En el último tercio de gestación las hembras gestantes tienen una disminución en el consumo de forraje (Joy et al. 2014, Nielsen et al. 2015), lo cual se soluciona suministrando una mayor cantidad de suplementos altos en energía (Giacomo et al. 2004).

La utilización de suplementos esta dado en base a su aporte nutricional y a la zona donde se produce, para el caso del estado de San Luis Potosí, es común la alimentación con grano de maíz, heno de alfalfa, melaza, sorgo, y algunos aceites para el caso de alimentación energética (Ochoa y Urrutia 2007, Silva et al. 2014, Shimada 2017). La soya, semilla de girasol y linaza son utilizados como fuente de suplementación proteica, y como alternativa para la época de sequía son utilizados los suplementos con nitrógeno no proteico como la urea, pollinaza y la gallinaza (Ochoa y Urrutia 2007).

En ovejas de pelo alimentadas con pasto de corte se suplementaron con $500 \mathrm{~g}_{\text {día }}{ }^{-1}$, donde la pollinaza se racionó en un $30 \%$ del suplemento, se observó que las ovejas suplementadas mostraron mayor peso y éste varió menos en las hembras, y sus corderos tendieron a presentar mayores pesos al destete con $10.65 \mathrm{~kg}$ contra $10.22 \mathrm{~kg}$ en corderos destetados de hembras no suplementadas (Ríos et al. 2005). En corderos de pelo en engorda en pastoreo se utilizó un suplemento a base de pollinaza de dos fuentes de cama, donde los corderos suplementados con pollinaza mejoraron los indicadores productivos, donde la ganancia diaria de peso fue de 101 a $107 \mathrm{~g} \mathrm{día}^{-1}$ (Ortíz et al. 2009).

La adición de zeolita en dietas para ovinos pelibuey canulados fueron estudiadas por Ruiz et al. (2008), a razón de 0, 1.5, 3.0 y 4.5\% en la dieta, no observó diferencias en la digestibilidad de la materia seca (67.9 a 70.8\%), materia orgánica (72.8 a 79.7\%) y fibra detergente ácida (32.2 a 33.4\%), pero si observaron mayor consumo de fibra detergente ácido digestible. Considerando todo lo anterior y en busca de hacer más productivo el sistema de producción de ovinos se planteó el objetivo de evaluar el comportamiento productivo de ovejas en pastoreo con y sin suplementación en épocas críticas y el comportamiento del peso al nacimiento y destete de los corderos en la lactancia. 


\section{MATERIALES Y MÉTODOS}

\section{Localización}

La investigación se realizó en la Facultad de Agronomía y Veterinaria de la Universidad Autónoma de San Luis Potosí, ubicada en el Ejido Palma de la Cruz, Municipio de Soledad de Graciano Sánchez, SLP, en el km. 14.5 de la Carretera San Luis PotosíMatehuala, que se ubica a $22^{\circ} 13^{\prime} 39.8^{\prime \prime} \mathrm{LN}$ y $100^{\circ}$ 50' 58.3" LO a 1835 msnm. El clima es seco (BS1) el menos seco de los secos. La temperatura media anual es de $17.1^{\circ} \mathrm{C}$. La precipitación pluvial anual es de 362 mm (García 2004).

\section{Animales}

Se utilizaron 24 ovejas vacías de la raza Rambouillet, con un peso promedio de $55 \mathrm{~kg}$ con edad promedio mayor a dos años. También, se usaron dos machos de la misma raza con edad promedio de dos años, uno como borrego celador y otro como reproductor.

\section{Tratamientos y diseño experimental}

El estudio se realizó en 2016, las hembras se distribuyeron al azar en tres grupos de 8 animales cada uno con los siguientes tratamientos: $\mathrm{T} 1$ = testigo que fueron hembras alimentadas solo con el pastoreo de una pradera de festuca, $\mathrm{T} 2$ = hembras alimentadas con pastoreo de una pradera de festuca más suplemento a base de un $85 \%$ de rastrojo, $10 \%$ de gallinaza y $5 \%$ de zeolita y $\mathrm{T}=3$ hembras alimentadas con pastoreo de una pradera de festuca más suplemento a base de un $75 \%$ de rastrojo, $20 \%$ de gallinaza y $5 \%$ de zeolita. Los ingredientes de las dietas se molieron en un molino de martillos y se mezclaron para hacer el suplemento de cada tratamiento, el cual se ofreció a las hembras por la tarde a las 15:00 h cuando se introducían a los corrales después del pastoreo.

\section{Manejo general de las hembras}

Durante el periodo de adaptación de 15 días, las hembras se despezuñaron manualmente con un cuchillo con gancho y se esquilaron manualmente con tijeras de esquila durante las primeras horas del día para disminuir el estrés y no alterar sus horas de pastoreo. Se desparasitaron durante los días de adaptación previos al empadre donde se aplicó Ivermectina ${ }^{\circledR}$ de manera subcutánea a dosis de $0.2 \mathrm{mg} \mathrm{kg}^{-1}$ por vía subcutánea. Se realizó desparasitación externa, donde las hembras se bañaron por inmersión en una tina con 50 litros de agua con Asuntol ${ }^{\circledR}$ Líquido $20 \%$ a razón de 10 $\mathrm{mL}$ en 10 litros de agua. Se vacunó contra brúcela con Biobac ${ }^{\circledR} 11$ vías, en una dosis de $2.5 \mathrm{~mL}$ por animal por vía subcutánea. También se aplicó vitaminas ADE a las ovejas a dosis de $3 \mathrm{~mL}$ por $50 \mathrm{~kg}$ por vía intramuscular. Se lavaron los bebederos y comederos cada tercer día, y se realizó limpieza de corrales cada 15 días.

\section{Manejo de los corderos}

Se desinfectó el ombligo de los corderos inmediatamente después del nacimiento con solución yodada, se registró la fecha de nacimiento, peso, sexo y el número de identificación de la madre en una bitácora. Los corderos se descolaron con el método de ligado, colocándola a los tres días de nacimiento y se aretaron a partir de los 15 días de nacimiento, también se aplicó $0.5 \mathrm{~mL}$ de vitamina $\mathrm{E}$ con selenio.

\section{Manejo reproductivo}

En los meses de agosto a septiembre se colocó un dispositivo intravaginal con $0.3 \mathrm{~g}$ de progesterona (CIDR) pfizer ${ }^{\circledR}$ a las 24 hembras en tratamiento para la sincronización de los estros, con una diferencia de 10 días por tratamiento y se retiró a los 9 días de su colocación. Después de $12 \mathrm{~h}$ del retiro del CIDR se introdujo un macho celador para detección de calores, ya identificadas las hembras que entraron en calor fueron introducidas con el macho reproductor 5 días en cada tratamiento. Se realizó diagnóstico de gestación a los 30 días después de la monta, con el equipo de ultrasonografía rectal modelo B Mode.

\section{Manejo nutricional}

Para la suplementación se tuvo un periodo de adaptación de 15 días antes de iniciar la colocación del CIDR de cada grupo, iniciando con un 
ofrecimiento del $50 \%$ de la cantidad del suplemento y se fue aumentando gradualmente hasta dar el $100 \%$ del suplemento a los ocho días de iniciado del empadre. Las hembras de los tres tratamientos se pastorearon en el mismo potrero previa estimación del forraje disponible de 8 a $14 \mathrm{~h}$ todos los días y posteriormente por la tarde fueron alojadas en corrales separados por tratamiento provistos de sombra (30\% del área), bebedero con agua limpia a libre acceso y comedero para ofrecer el suplemento correspondiente al tratamiento. La suplementación fue suministrada solamente en las siguientes épocas: antes y durante el empadre 15 días, 40 días del último tercio de gestación y durante dos meses de lactancia exclusivamente a los tratamientos 2 y 3 .

En el tratamiento 2 se ofreció $300 \mathrm{gr} \mathrm{día}^{-1}$ de suplemento y $400 \mathrm{~g} \mathrm{día}^{-1}$ para el tratamiento 3 por animal. Considerando los aportes nutricionales de los ingredientes utilizados y la cantidad ofrecida se calculó el aporte del suplemento que fue de $41 \mathrm{~g}$ de PC kg ${ }^{-1}$ de MS para el tratamiento 2 y $74.6 \mathrm{~g}$ de PC $\mathrm{kg}^{-1}$ de MS para el tratamiento 3 por animal por día y $0.469 \mathrm{Mcal} \mathrm{kg}^{-1}$ de MS por animal para el T2 y 0.689 Mcal kg ${ }^{-1}$ de MS por animal para el T3 (Tabla 1).

La pradera fue de pasto festuca (Festuca arundinaceae) con dos años de edad, la cual se pastoreó antes de la floración con 15.95\% PC (Análisis de laboratorio) y $2.366 \mathrm{Mcal}$ (Mieres 2004). Para el manejo del pastoreo se cercó la pradera con malla borreguera y se dividió en cuatro potreros, los cuales se manejaron en un sistema rotacional con periodo de pastoreo de 10 días promedio. Los riegos se dieron cada 20 días en promedio con riego por gravedad con el método de melgas.

\section{Variables evaluadas}

Peso al empadre (PE): las hembras se pesaron en el último día de la suplementación del empadre con una báscula electrónica marca Torrey ${ }^{\circledR}$, modelo FS-250/50, con capacidad de $250 \mathrm{~kg}$ y una aproximación de $0.05 \mathrm{~kg}$. Peso al parto (PP): las hembras se pesaron con báscula electrónica marca Torrey ${ }^{\circledR}$ modelo FS-250/50, posterior de haber arrojado la pla- centa completamente. Peso al destete (PD): las hembras se pesaron con báscula electrónica marca Torrey ${ }^{\circledR}$ modelo FS-250/50, 60 días posteriores al parto. Condición corporal al empadre (CCE): se realizó mediante la palpación en las ovejas en las apófisis espinosas y transversas de la columna vertebral, al momento del empadre. Se registró de la siguiente manera: condición 1 emaciación, condición 2 delgado, condición 3 bueno, condición 4 gordo, condición 5 obeso. Condición corporal al parto (CCP): mediante la técnica empleada para condición corporal al empadre. Condición corporal al destete (CCD): mediante la técnica empleada para condición corporal al empadre. Peso al nacimiento del cordero (PNC): se midió inmediatamente al ser expulsado por la madre antes del consumo del calostro, con báscula electrónica marca Torrey ${ }^{\circledR}$ modelo FS-50/5, con capacidad de $50 \times 0.005 \mathrm{~kg}$. Peso al destete de corderos (PDC): se realizó a los 60 días de nacimiento con una báscula electrónica marca Torrey ${ }^{\circledR}$ modelo FS-50/5, con capacidad de $50 \times 0.005 \mathrm{~kg}$. Ganancia de Peso de Hembras (GPH): los periodos evaluados fueron Empadre:Parto (GPHEP), Parto:Destete (GDHPD) y Empadre:Destete (GPHED), las ganancias de peso se calcularon por diferencia de peso de los valores obtenidos al inicio del empadre (IE), al momento del parto (MP) y final del destete (FD), y se utilizaron las siguientes fórmulas: MP-IE, FD-MP y FD-IE. Ganancia diaria de peso de corderos (GDPC): se pesó a los corderos cada 15 días desde su nacimiento y hasta el destete y se utilizó la siguiente fórmula para obtener la ganancia diaria de peso. Peso final - Peso Inicial/15 días.

\section{Análisis estadístico}

Para el análisis de los datos se utilizó un modelo estadístico completamente al azar con ocho repeticiones, donde la unidad experimental fue la oveja y los corderos. Los datos obtenidos se analizaron mediante el paquete estadístico SAS ${ }^{\circledR}$ versión 9.3 y se realizó la prueba de Tukey al $\mathrm{P}<$ 0.05 para la comparación de medias (SAS 2011). 
Tabla 1. Aporte de nutrientes por el suplemento ofrecido en los tratamientos.

\begin{tabular}{|c|c|c|c|c|c|c|}
\hline & \multirow[b]{2}{*}{ Ingrediente } & \multirow[b]{2}{*}{$\begin{array}{l}\text { MS } \\
(\mathrm{kg})\end{array}$} & \multicolumn{2}{|c|}{ Aporte de nutrientes $1 \mathrm{~kg} \mathrm{MS}$} & \multicolumn{2}{|c|}{ Aporte por ración } \\
\hline & & & $\begin{array}{c}P C \\
(\% / \mathrm{kg} / \mathrm{MS})\end{array}$ & $\begin{array}{c}\mathrm{EM} \\
\text { (Mcal/kg/MS) }\end{array}$ & $\begin{array}{l}\mathrm{PC} \\
(\%)\end{array}$ & $\begin{array}{l}\text { EM } \\
\text { (Mcal) }\end{array}$ \\
\hline \multirow[t]{4}{*}{$\mathrm{T} 1$} & Rastrojo de maíz & 0 & 0 & 0 & 0 & 0 \\
\hline & Pollinaza & 0 & 0 & 0 & 0 & 0 \\
\hline & Zeolita & 0 & 0 & 0 & 0 & 0 \\
\hline & Total & 0 & 0 & 0 & 0 & 0 \\
\hline \multirow[t]{4}{*}{ T2 } & Rastrojo de maíz* & 0.15 & 4.9 & 1.66 & 0.74 & 0.249 \\
\hline & Pollinaza** & 0.10 & 33.6 & 2.20 & 3.36 & 0.220 \\
\hline & Zeolita & 0.05 & - & - . & - & - \\
\hline & Total & 0.30 & & & 4.1 & 0.469 \\
\hline \multirow[t]{4}{*}{ T3 } & Rastrojo de maíz & 0.15 & 4.9 & 1.66 & 0.74 & 0.249 \\
\hline & Pollinaza & 0.20 & 33.6 & 2.20 & 6.72 & 0.440 \\
\hline & Zeolita & 0.05 & - & - & - & - \\
\hline & Total & 0.40 & & & 7.46 & 0.689 \\
\hline
\end{tabular}

MS = Materia Seca, PC = Proteína Cruda, EM = Energía Metabolizable. Fuente: ${ }^{* * T o b i a ~}$ y Vargas (2000), Fuentes et al. (2001).

\section{RESULTADOS}

\section{Comportamiento del peso de las hembras}

EI PE mostró diferencias significativas $(p<$ 0.05 ) entre tratamientos (Tabla 2), donde el T2 fue el que obtuvo los mayores PE con $54.2 \mathrm{~kg}$, y el T3 el menor PE con $49.2 \mathrm{~kg}$. En cuanto al PP se obtuvieron diferencias significativas $(p<0.05)$ entre los tratamientos (Tabla 2), donde se observa que los mayores PP lo obtuvieron el T2 y el T3 con 71.9 y $69.4 \mathrm{~kg}$, respectivamente, y el menor valor los mostró el T1 con $61.3 \mathrm{~kg}$. Las hembras que recibieron suplementación ganaron mucho más pesos que las que no recibieron el suplemento, e incluso el T3 mostró mayor peso al parto que el que mostraba en el empadre porque fue el grupo que presentaba menor peso al empadre.

Tabla 2. Peso y condición corporal del empadre, al parto y al destete; de ovejas rambouvillet en pastoreo con festuca suplementadas con rastrojo, gallinaza y zeolita.

\begin{tabular}{crrrrrr}
\hline Tratamiento & $\mathrm{PE}$ & $\mathrm{PP}$ & $\mathrm{PD}$ & $\mathrm{CCE}$ & $\mathrm{CCP}$ & $\mathrm{CCD}$ \\
\hline $\mathrm{T} 1$ & $51.8^{a b}$ & $61.3^{b}$ & $51.8^{b}$ & $2.8^{a}$ & $3.5^{a}$ & $2.0^{b}$ \\
$\mathrm{~T} 2$ & $54.2^{a}$ & $71.8^{a}$ & $70.4^{a}$ & $2.9^{a}$ & $3.7^{a}$ & $2.7^{a}$ \\
$\mathrm{~T} 3$ & $49.2^{b}$ & $69.4^{a}$ & $68.4^{a}$ & $2.5^{a}$ & $3.5^{a}$ & $3.0^{a}$ \\
Media & 52.4 & 67.8 & 64.9 & 1.4 & 1.4 & 1.6 \\
\hline
\end{tabular}

Letras distintas ${ }^{a, b, c \ldots}$ en las columnas indican diferencia significativa $(p<0.05) . P E=$ Peso al Empadre, $P P=$ Peso al Parto, $P D=$ Peso al destete, $\mathrm{CCE}=$ Condición corporal al empadre, $\mathrm{CCP}=$ Condición corporal al parto, $\mathrm{CCD}=$ Condición corporal al destete.
Para la variable PD se obtuvieron diferencias significativas $(p<0.05)$ entre tratamientos (Tabla 2$)$, siendo los T2 y T3 los de mayor valor con 70.4 y 68.4 $\mathrm{kg}$, respectivamente, y el T1 el de menor peso. Lo que permite observar que las hembras suplementadas en la lactancia mantienen el peso que mostraron al parto.

\section{Comportamiento de la condición corporal de las hembras}

Para CCE, CCP y CCD, solamente se observaron diferencias significativas $(p<0.05)$ para CCD entre tratamientos (Tabla 2), donde la mejor CCD la obtuvieron los tratamientos T2 y T3 con 2.7 y 3.0, respectivamente.

\section{Ganancia de peso de las hembras}

Para la ganancia de peso de hembras se observaron diferencias significativas $(p<0.05)$ para los tres periodos evaluados (GPHEP, GPHPD y GPHED; Tabla 3), donde es factible observar que para GPHEP las mayores GDH fueron para T2 y T3 con valores de 17.7 y $20.9 \mathrm{~kg}$, respectivamente, ganados en el periodo.

\section{Comportamiento del peso de los corderos}

Para PNC solo se observaron diferencias significativas $(p<0.05)$ entre el tratamiento T3 contra el T1 y T2, siendo éstos dos últimos iguales (Tabla 4), de lo cual se puede observar el mayor valor para T3 con $5.429 \mathrm{~kg}$, lo cual demuestra que el nivel 
de alimentación de la madre impacta en el peso al nacimiento.

Tabla 3. Ganancia de peso de hembras de ovejas rambouvillet en pastoreo de festuca y suplementadas con rastrojo, pollinaza y zeolita.

\begin{tabular}{crrr}
\hline Tratamiento & GDPHP $(\mathrm{kg})$ & GDPHD $(\mathrm{kg})$ & GPHED $(\mathrm{kg})$ \\
\hline T1 & $9.442^{b}$ & $-9.417^{b}$ & $0.03^{c}$ \\
T2 & $17.700^{a}$ & $-1.429^{a}$ & $16.27^{b}$ \\
T3 & $20.188^{a}$ & $-0.938^{a}$ & $19.25^{a}$ \\
Media & 12.888 & -3.523 & 12.76 \\
\hline
\end{tabular}

Letras distintas $a, b, c \ldots$ en las columnas indican diferencia significativa $(p<0.05)$. GDPHP = Ganancia de Peso del Periodo al Parto, GDPHD $=$ Ganancia de Peso del Periodo del Parto al Destete, GDPHEP = Ganancia de Peso Periodo del Empadre al Destete.

EI PDC se vio afectado significativamente $(p$ $<0.05$ ) por la alimentación de las ovejas (Tabla 4), ya que el T3 mostró el mayor peso al destete de los corderos con $20.260 \mathrm{~kg}$ (Tabla 1), a la cual le siguió el T2 con $18.150 \mathrm{~kg}$ al destete que recibió un nivel menor de proteína y energía que el T3 en la suplementación. En cambio, el T1 recibió menor proteína y energía, ya que no recibió suplemento y por lo tanto obtuvo el menor peso a destete $(15.871 \mathrm{~kg})$, lo cual demuestra que el nivel de alimentación de la madre impacta en el peso al destete.

\section{Ganancia diaria de peso de corderos}

La GDPC mostró diferencias significativas ( $p$ $<0.05$ ) entre tratamientos (Tabla 4), donde el T3 y T2 mostraron los mayores valores con 249 y 230 gr/día, respectivamente, en cambio el T1 mostró las menores GDP con $189 \mathrm{~g} \mathrm{día}^{-1}$.

\section{DISCUSIÓN}

\section{Comportamiento del peso de las hembras}

Para PE existen estudios en los que se ha encontrado que este tipo de suplemento se ha utilizado antes y durante el empadre para mejorar parámetros productivos y reproductivos, quienes observaron pesos finales al final de la suplementación de $33.1 \pm$ $1.7 \mathrm{~kg}$, utilizando un suplemento energético durante 28 días antes del empadre en ovejas de pelo en pastoreo en vegetación nativa durante 5 horas diarias (Cansino-Arroyo et al. 2009).
Para PP los datos obtenidos fueron mayores a los observados por Rueda y De Combellas (1999), cuando estudiaron ovejas criollas $(29.0 \mathrm{~kg})$ al no utilizar suplemento en los últimos días de gestación, y a los de McWilliam et al. (2005) que fueron de 57.5 kg en ovejas de la raza Romney en Nueva Zelanda, suplementadas durante el empadre y en el último tercio de gestación. Estos datos pueden estar influenciados por la alimentación recibida por los animales, ya que el nivel nutricional de las madres durante la gestación puede afectar el desempeño productivo y reproductivo de la descendencia (Martin et al. 2006). Datos semejantes fueron obtenidos por Rivas-Jacobo et al. (2018) bajo el mismo modelo de evaluación con suplementación con pesos promedio de $66.30 \mathrm{~kg}$ al parto cuando las hembras fueron pastoreadas en pastizales nativos suplementadas con rastrojo, maíz, pasta de soya y glicerina. Para Vicente-Pérez et al. (2015), al suplementar energéticamente ovejas en el último tercio de gestación se mejoró el peso de las hembras al parto.

Para la variable PD al observar los datos es factible identificar que los tratamientos con suplemento (mayor porcentaje de proteína en la dieta) mantuvieron su peso obtenido al parto y mejoraron los pesos obtenidos al empadre, en cambio en el T1 sin suplemento no mantuvo su peso al parto pero si su peso obtenido en el empadre, lo cual también es bueno y pudo deberse a que la alimentación recibida por la pradera fue buena, ya que la festuca ofreció un porcentaje de proteína adecuado para las funciones reproductivas y productivas de las hembras en pastoreo y no fueron afectadas en mayor grado como cuando pierden peso, ya que normalmente en fases productivas de la lactación se elevan las necesidades nutricionales de la oveja. En esta investigación se observa que las hembras suplementadas ganaron peso y las que no recibieron suplementación perdieron peso, datos semejantes observaron RivasJacobo et al. (2018) para las hembras que fueron suplementadas que ganaron peso, mientras las que no se suplementaron perdieron peso, lo que perjudicará a las hembras en su retorno al celo y próximo ciclo reproductivo. Los mejores pesos al destete que se obtuvieron fueron las hembras suplementadas 
Tabla 4. Peso al nacimiento y al destete, ganancia diaria de peso de corderos rambouvillet lactantes en pastoreo con festuca con y sin suplementación.

\begin{tabular}{crrr}
\hline Tratamiento & Peso al Nacimiento $(\mathrm{kg})$ & Peso al Destete $(\mathrm{kg})$ & Ganancia Diaria de Peso $(\mathrm{gr})$ \\
\hline T1 & $4.386^{b}$ & $15.871^{c}$ & $189^{b}$ \\
T2 & $4.657^{b}$ & $18.150^{b}$ & $230^{a}$ \\
T3 & $5.429^{a}$ & $20.260^{a}$ & $249^{a}$ \\
Media & 4.824 & 18.09 & 222 \\
\hline Letras distintas $^{a, b, c \ldots .}$ en las columnas indican diferencia significativa $(\mathrm{p}<0.05)$.
\end{tabular}

con Rastrojo, Gallinaza y Zeolita, cuestión que puede deberse a una mejor condición corporal de las hembras por una mejor eficiencia alimenticia como lo menciona Estrada-Ángulo et al. (2017), quienes incluyeron de zeolita por arriba de $1.5 \%$ en corderos en finalización. Algo similar observó Vicente-Pérez et al. (2015), al suplementar energéticamente ovejas en el último tercio de gestación quienes señalaron que se mejoró el consumo de energía y el peso de las hembras.

\section{Comportamiento de la condición corporal de las hembras}

En lo que respecta a la CCD de los T2 y T3, los datos demuestran que las hembras suplementadas $y$ no suplementadas mantienen su condición corporal al momento del parto, mientras que cuando entran a la etapa productiva o la lactancia pueden cambiar su condición corporal si se descuida su alimentación, observando de esta manera que las hembras suplementadas mantuvieron su condición corporal al destete. Los valores obtenidos de CCD son aceptables, lo cual se debe a la buena alimentación de las hembras por el suplemento recibido y que no permitieron gran movilización de reservas corporales al comparar los pesos iniciales al empadre y lo ganado al parto, en cambio el T1 el valor de CC se considera delgado, lo cual es malo porque se permitió remover mayores reservas corporales al proporcionarle menores nutrimentos en la alimentación. Valores semejantes fueron observados por Rivas-Jacobo et al. (2018) al realizar suplementación bajo el mismo patrón de esta investigación, donde se utilizó suplementos a base de rastrojo + maíz molido, y rastrojo + pasta de soya y glicerina, observando CCP de 3.5. En cambio Estrada-Ángulo et al. (2017) estudiaron cuatro niveles de inclusión de zeolita $(0,1.5,3.0$ y $4.5 \%)$ en la dieta de ovinos de pelo y observaron que sustituir maíz y pasta de soja por zeolita hasta por encima de $1.5 \%$ se observaron resultados, siendo que la respuesta máxima para el nivel de inclusión de 3.0\% mejoró $(p<0.01)$ en $6.8 \%$ la eficiencia alimenticia, en $8.4 \%$ la utilización de la energía neta de la dieta y en $8.9 \%$ la retención aparente de energía por unidad de MS consumida, lo que se refleja en una mejor condición corporal de las hembras. Datos similares observados por Vicente-Pérez et al. (2015), al suplementar energéticamente ovejas en el último tercio de gestación se mejoró el consumo de energía, el peso de las hembras al parto y la condición corporal.

\section{Ganancia de peso de las hembras}

Para GPHPD aunque hubo pérdidas de peso fueron significativamente menores en los T2 y T3, reflejada de igual manera a la mejor alimentación recibida. En GPHEP la mejor ganancia de peso fue para T3 con $19.25 \mathrm{~kg}$, seguida del T2 con $16.27 \mathrm{~kg}$, aspecto que demuestra una buena alimentación de las hembras durante las épocas críticas de mayor demanda de nutrimentos por concepto de gestación y producción de leche para la alimentación del cordero, ya que esto asegura que las hembras lleguen al destete con buen peso y condición corporal y estén aptas para la siguiente etapa reproductiva y retorno al estro, y con esto asegurar una excelente comportamiento reproductivo y reproductivo. Aspecto que puede estar relacionado con la adición de zeolita en la dieta, ya que Estrada-Ángulo et al. (2017), observaron que la inclusión de zeolita por arriba de $1.5 \%$ a la dieta de finalización en corderos de pelo mejora la utilización de la energía neta de la dieta. En base al rendimiento productivo y la eficiencia en la utilización de la energía neta de la dieta, la respuesta óptima observada para los corderos fue con el $3 \%$ de in- 
clusión de zeolita. Rivas-Jacobo et al. (2018) bajo el mismo modelo de evaluación hembras criollas encastadas de rambouvillet y suffolk fueron pastoreadas en pastizales nativos suplementadas con rastrojo, maíz, pasta de soya y glicerina observaron valores de ganancia de peso de $5.66 \mathrm{~kg}$ en hembras con suplementación y pérdidas de peso de $-1.07 \mathrm{~kg}$ en hembras sin suplementación. En cambio Vicente-Pérez et al. (2015), al suplementar energéticamente ovejas en el último tercio de gestación señalaron que se mejoró la ganancia de peso y el peso de las hembras.

\section{Comportamiento del peso de los corderos}

Los datos obtenidos para PNC fueron similares observados por Forero et al. (2017), quienes obtuvieron pesos de $5.31 \mathrm{~kg}$ para machos de nacimientos sencillos. Se sabe que la alimentación de las borregas antes y después del parto influye indirectamente sobre la salud y desarrollo de las crías (Chávez et al. 1995), lo cual concuerda con Forero et al. (2017), quienes observaron en ovinos que es necesario una modulación adecuada de la alimentación de las ovejas durante el último tercio de la gestación para paliar el efecto de las altas temperaturas sobre el peso de los corderos al nacimiento. Resultados semejantes los obtuvieron Freitas-De-Melo et al. (2015), quienes reportaron pesos al nacimiento de los corderos de $4.8 \pm 0.2 \mathrm{~kg}$, al igual que Rivas-Jacobo et al. (2018) bajo el mismo modelo de evaluación de hembras criollas encastadas de rambouvillet y suffolk fueron pastoreadas en pastizales nativos suplementadas con rastrojo, maíz, pasta de soya y glicerina observaron valores de PN de $4.8 \mathrm{~kg}$. Pero resultados mayores a los observados por Catalán et al. (2018) en ovejas criollas cruzadas con Dorper, Black Belly, Pelibuey y Kathadin pesos al nacimiento de corderos de $3.19 \mathrm{~kg}$ en partos simples que provenían de hembras bajo pastoreo en pasturas nativas con pasto, arbustos y árboles de la región.

Los datos obtenidos de PDC en esta investigación para las hembras suplementadas fueron mayores a los obtenidos por Forero et al. (2017), pero semejantes al T1 sin suplementar, que fue de 16.265 $\mathrm{kg}$ en promedio para corderos destetados a los 45 días. En cambio, Castellaro et al. (2015) observó pesos similares a los obtenidos en esta investigación, tanto para hembras como para machos con pesos de 18.59 y $18.80 \mathrm{~kg}$, respectivamente provenientes de hembras de las razas Merino precoz, Suffolk y Mestizos destetados a los 60 días bajo pastizales naturales en zonas semiáridas; al igual que González-Anaya et al. (2013), quienes observaron pesos promedio al destete de $17.9 \mathrm{~kg}$, donde los corderos nacidos como sencillos fueron más pesados (19.6 kg.), que los nacidos como dobles (16.5 kg.) en un sistema estabulado donde las hembras de la raza Rambouillet recibieron una ración con $14 \%$ de $\mathrm{PC}$ y los corderos recibieron una ración con $16 \%$ de PC y $70 \%$ de TND destetados a 84 días de edad.

\section{Ganancia diaria de peso de corderos}

La GDPC se debió a la mejor alimentación de las hembras al recibir además de la materia seca y nutrimentos de la pradera un suplemento proteico a base de rastrojo, pollinaza y zeolita. Datos semejantes a los obtenidos por con Forero et al. (2017), para la raza Merino $x$ Merino (236 $\mathrm{g} \mathrm{día}^{-1}$ ), Merino Precoz x Merino (232 $\mathrm{g} \mathrm{día}^{-1}$ ) y para lle de Francia $x$ Merino (268 $\mathrm{g} \mathrm{día}^{-1}$ ) que provenían de ovejas alimentadas con paja de cebada ad libitum y $0.9-1.0 \mathrm{~kg}$ de un concentrado comercial, que contenía un $15 \%$ de proteína bruta y cuyos componentes principales fueron cebada, torta de girasol, pulpa de remolacha, melaza y corrector vitamínico-mineral; al igual a los de González-Anaya et al. (2013), quienes observaron ganancias diarias de peso promedio de $235 \mathrm{gr} / \mathrm{día}$ en corderos provenientes de hembras de la raza Rambouillet.

\section{CONCLUSIONES}

El peso y la condición corporal de hembras al destete estuvo influenciada por el suplemento estratégico a base de rastrojo, gallinaza y zeolita que se vio reflejado en una mejor producción de las hembras y corderos. La ganancia de peso en las hembras fue favorecida por la suplementación estratégica a base de rastrojo, gallinaza y zeolita por un mayor aporte de nutrimentos durante la etapa de empadre, último tercio de gestación y lactación. La mejor productividad 
de las hembras fue influenciada por el mayor nivel de proteína y energía recibido en la dieta, lo que aseguró mayor ganancia de peso y condición corporal de las hembras. El peso al nacimiento y peso al destete de los corderos fue favorecido por la suplementación es- tratégica al mantener en mejores condiciones corporales a las madres, asegurando de esta manera una mejor alimentación del cordero al desarrollar mejores ganancias de peso.

\section{LITERATURA CITADA}

Ben SH, Nefzaoui A (2003) Review, feed blocks as alternative supplements for sheep and goats. Small Ruminant Research 49: 275-288.

Cansino-Arroyo J, Herrera-Camacho J, Aké-López JR (2009) Tasas de concepción, fertilidad y prolificidad en ovejas de pelo alimentadas con dietas enriquecidas con ácidos grasos poliinsaturados. Universidad y Ciencia 25: 181-185.

Castellaro GG, Ximena GF, Magofke SJC, Marín FG (2015) Peso vivo y crecimiento de corderos merino precoz, suffolk y mestizos en praderas mediterráneas semiáridas de chile. Chilean Journal Agricultural \& Animal Science 32: 60-69.

Catalán A, Hernández A, Fraga LM, Mireles EJ (2018) Efectos no genéticos en el peso al nacer de ovejas criollas MEVEZUG en Guerrero, México. Cuban Journal Agricultural Science 52: 117-125.

Chávez GR, Castellanos RAF, Velázquez MPA (1995) Producción de las ovejas pelibuey pre y posparto alimentadas con diversos aportes nutricionales. Técnica Pecuaria en México 33: 183-191.

Estrada-Ángulo A, Coronel-Burgos F, Castro PBI, López SMA, Barreras A, Angulo-Montoya C, Contreras PG, Plascencia A (2017) Efecto de la inclusión de zeolita (clinoptilolita) en ovinos en etapa de finalización: Respuesta productiva y energética de la dieta. Archivos de Zootecnia 66: 381-386.

Forero FJ, Venegas M, Alcalde MJ, Daza A (2017) Peso al nacimiento y al destete y crecimiento de corderos Merinos y cruzados con Merino Precoz y lle de France: Análisis de algunos factores de variación. Archivos de Zootecnia 66: 89-97.

Freitas-De-Melo A, Ungerfeld R, Hötzel MJ, Abud MJ, Alvarez-Oxiley A, Orihuela A, Damian JP, Pérez-Clariget $R$ (2015) Mother-young behaviours at lambing in grazing ewes: effects of lamb sex and food restriction in pregnancy. Applied Animal Behaviour Science 168: 31-36.

Fthenakis GC, Arsenos G, Brozos C, Fragkou IA, Giadinis ND, Giannenas I, Mavrogianni VS, Papadopoulos E, Valasi I (2012) Health management of ewes during pregnancy. Animal Reproduction Science 130: 198-212.

Fuentes J, Magaña C, Suárez L, Peña R, Rodríguez S, Ortíz De la RB (2001) Análisis químico y digestibilidad "In vitro" del rastrojo de maíz (Zea mays L.). Agronomía Mesoamericana 12: 189-192.

Galaviz-Rodríguez JR, Vargas-López S, Zaragoza-Ramírez JL, Bustamante-González A, Ramírez-Bribiesca E, Guerrero-Rodríguez JD, Hernández ZJS (2011) Evaluación territorial de los sistemas de producción ovina en la región nor-poniente de Tlaxcala. Revista Mexicana de Ciencias Pecuarias 2: 53-68.

García AE (2004) Modificaciones al sistema de clasificación climática de Koppen. Quinta Edición. Instituto Nacional de Geografía. UNAM. México. 246p.

Giacomo RSP, Enne G, Ligios S, Molle G (2004) Nutrition and reproduction. In: Pulina G, Bencini R (eds) Dairy sheep nutrition. CABI Publishing, Wallingford. UK. pp: 109-128. 
González-Anaya A, Ochoa-Cordero MA, Torres-Hernández G, Díaz-Gómez MO, González-Camacho JM (2013) Influencia de factores ambientales en el comportamiento productivo en fase predestete de corderos rambouillet. Abanico Veterinario 3: 31-38.

Grajales LH, Moreno DC, Cárdenas RE (2011) Guía técnica de producción ovina y caprina: IV. Aspectos de manejo y control nutricional y alimenticio. Primera Edición. Universidad Nacional de Colombia. Internacional Print Ltda. Colombia. 62p.

Hauken P, Williman M, Milton J, Kelly R, Nowak R, Blache B (2012) Nutritional supplementation during the last week of gestation increased the volume and reduced the viscosity of colostrum produced by twin bearing ewes selected for nervous temperament. Small Ruminant Research 105: 308-314.

Joy M, Ripoll-Bosch R, Sanz A, Molino F, Blasco I, Álvarez-Rodríguez J (2014) Effects of concentrate supplementation on forage intake, metabolic profile and milk fatty acid composition of unselected ewes raising lambs. Animal Feed Science and Technology 187: 19-29.

Martin GB, Kadowa H (2006) "Clean, green and ethical" animal production. Case study: Reproductive efficiency in small rumiants. Journal of Reproduction and Development 52: 145-152.

Mcwilliam E, Barry T, López-Villalobos N, Cameron P, Kemp P (2005) Effects of willow (Salix) versus poplar (Populus) supplementation on the reproductive performance of ewes grazing low quality drought pasture during mating. Animal Feed Science And Technology 119: 69-86.

Mieres JM (2004) Guía para la alimentación de rumiantes. Programa Nacional Bovinos para Leche. INIA La Estanzuela. Serie Técnica $N^{\circ} 142$. INIA. Editorial Hemisferio Sur. Uruguay. 84p.

Nielsen M, Nadeau E, Markussen B, Helander C, Eknæs M, Nørgaard P (2015) Relationship between energy intake and chewing index of diets fed to pregnant ewes. Small Ruminant Research 130: 108-116.

Ortíz A, Elías A, Valdivié M (2009) Utilización de diferentes fuentes de pollinaza como complemento alimenticio en la ceba de ovinos en pastoreo. Revista Cubana de Ciencia Agrícola 43: 245-249.

Rivas-Jacobo MA, Santillán-Cortina E, Herrera-Corredor CA, Villarreal-González JA, Hernández-Garay $\mathrm{A}^{\dagger}$, Romero-Dávila A, Joaquín-Cancino S (2018) Productividad de ovejas criollas bajo pastoreo extensivo suplementadas energéticamente en época crítica. Agroproductividad 11: 100-106.

Rosales NC, Meza-Herrera C, Morón-Cedillo F, Flores-Nájera M, Gámez-Vázquez H, Cuevas-Reyes V, Liu S (2015) Effects of vitamin E supply during late gestation and early lactation upon colostrum composition, milk production and quality in nutritional restricted ewes. Small Ruminant Research 133: 77-81.

Rueda E, De Combellas J (1999) Evaluación de la suplementación con bloques multinutricionales en un sistema de producción ovina, ovejas en lactancia. Revista de la Facultad de Agronomía (LUZ) 16: 79-88.

Ruíz O, Castillo Y, Elías A, Arzola C, Rodríguez C, Salinas J, La OO, Holguín C (2008) Efecto de cuatro niveles de zeolita en la digestibilidad y consumo de nutrientes en ovinos alimentados con heno de alfalfa y concentrado. Revista Cubana de Ciencia Agrícola 42: 367-370.

SAS Institute Inc (2011) Base SAS ${ }^{\circledR}$ 9.3 Procedures Guide: Statistical Procedures. SAS Institute Inc., Cary, NC, USA. 527p.

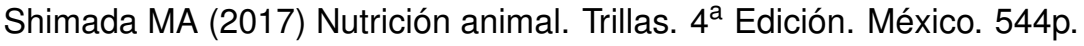

Silva VO, Lopes E, Andrade EF, Sousa RV, Zangeronimo MG, Pereira LJ (2014) Use of biodiesel co-products (Glycerol) as alternative sources of energy in animal nutrition: a systematic review. Archivos de Medicina Veterinaria 46: 111-120. 
Tobia C, Vargas E (2000) Evaluación de las excretas de pollos de engorde (Pollinaza) en la alimentación animal. II. Fraccionamiento de los components nitrogenados y contenido de energía. Agronomía Costarricence 24: 55-62.

Vicente-Pérez R, Avendaño-Reyes L, Álvarez FD, Correa-Calderon A, Meza-Herrera CA, Mellado M, Quintero JA, Macías-Cruz U (2015) Comportamiento productivo, consumo de nutrientes y productividad al parto de ovejas de pelo suplementadas con energía en el preparto durante verano e invierno. Archivos de Medicina Veterinaria 47: 301-309. 